\title{
A KATO-YAU INEQUALITY AND DECAY ESTIMATE FOR EIGENSPINORS
}

\author{
PAUL M. N. FEEHAN
}

\section{INTRODUCTION}

The purpose of this article is to prove a Kato-Yau inequality for harmonic spinors and a decay estimate for eigenspinors. We also describe some new applications to gauge theoryspecifically, to estimates used when gluing and ungluing PU(2) monopoles [13], [16].

1.1. Statement of results. Let $(X, g)$ be an oriented, Riemannian, smooth four-manifold and let $\Omega=\Omega\left(x_{0}, r_{0}, r_{1}\right)$ denote the annulus $B\left(x_{0}, r_{1}\right)-\bar{B}\left(x_{0}, r_{0}\right)$, where $0<4 r_{0}<r_{1}<\infty$. Denote $r=\operatorname{dist}_{g}\left(x, x_{0}\right)$. Consider a triple $(X, \Omega, g)$ where the Laplacian $\Delta_{g}$ on $C^{\infty}(X)$ has a $C^{\infty}$ Green kernel $G_{g}$ and there is a constant $c_{0} \geq 1$ such that

$$
c_{0}^{-1} r^{-2} \leq G_{g}\left(x, x_{0}\right) \leq c_{0} r^{-2}, \quad x \in \Omega\left(x_{0}, r_{0}, r_{1}\right),
$$

and the scalar curvature, $\kappa_{g}$, of the Levi-Civita connection $\nabla_{g}$ obeys

$$
\left|\kappa_{g}\right|(x) \leq c_{1} \varepsilon r^{-2} \quad \text { and } \quad\left|\kappa_{g}\right|(x) \leq c_{2}\left(r_{0}^{2} r^{-4}+r_{1}^{-2}\right), \quad x \in \Omega\left(x_{0}, r_{0}, r_{1}\right) .
$$

Let $(\rho, W)$ be a $\operatorname{spin}^{c}$ structure [28] on $X$ with $C^{\infty}$ unitary connection $A_{d}$ on $\operatorname{det}\left(W^{+}\right)$and spin connection on $W=W^{+} \oplus W^{-}$, let $E$ be a Hermitian bundle over $X$, and let $A$ be an $C^{\infty}$ unitary connection on $E$. Assume that $A_{d}$ and $A$ are Yang-Mills connections whose curvatures obey, for some $\varepsilon>0$ to be specified,

$$
\left\|F_{A_{d}}\right\|_{L^{2}\left(\Omega\left(x_{0}, r_{0}, r_{1}\right)\right)} \leq \varepsilon \quad \text { and } \quad\left\|F_{A}\right\|_{L^{2}\left(\Omega\left(x_{0}, r_{0}, r_{1}\right)\right)} \leq \varepsilon
$$

Let $D_{A}$ be the Dirac operator, on sections of $V=W \otimes E$, defined by $\left(\rho, g, A_{d}, A\right)$.

Theorem 1.1. Continue the notation and assumptions of the preceding paragraph. Given positive constants $c_{0}, c_{1}, c_{2}$ and an integer $k \geq 0$, there are positive constants $\varepsilon\left(c_{0}, c_{1}, c_{2}\right) \leq 1$ (independent of $k$ ) and $c_{3}\left(c_{0}, c_{1}, c_{2}, k\right)$, with the following significance. If $0<4 r_{0}<r_{1}<\infty$ and $\phi \in L^{2}\left(\Omega\left(x_{0}, r_{0}, r_{1}\right), V\right)$ satisfies

$$
D_{A} \phi=0 \quad \text { on } \Omega\left(x_{0}, r_{0}, r_{1}\right) \text {, }
$$

and conditions (1.1), (1.2), and (1.3) hold, then for all $x \in \Omega\left(x_{0}, 2 r_{0}, r_{1} / 2\right)$,

$$
\left|\nabla_{A}^{k} \phi\right|_{g}(x) \leq c_{3} r^{-k}\left(r_{0} r^{-3}+r_{1}^{-2}\right)\|\phi\|_{L^{2}\left(\Omega\left(x_{0}, r_{0}, r_{1}\right), g\right)} .
$$

If $\phi$ is an eigenspinor of $D_{A}$, with non-zero eigenvalue $\mu$, then $\phi$ is harmonic with respect to the Dirac operator associated to the "Friedrich connection" [19] defined by the connection $A$ and eigenvalue $\mu$ (see $\$ 5.2$ ). Theorem 1.1 then leads to the

Date: This version: August 9, 2000. First version: March 2, 1999. math.DG/9903021.

The author was supported in part by NSF grant DMS 9704174 and, through the Institute for Advanced Study (1998-1999), by NSF grant DMS 9729992. 
Corollary 1.2. Continue the hypotheses of Theorem 1.1, except that we allow

$$
D_{A} \phi=\mu \phi \quad \text { on } \quad \Omega\left(x_{0}, r_{0}, r_{1}\right)
$$

for some $\mu \in \mathbb{R}$, instead of equation (1.4), and also assume

$$
r_{1}^{2} \leq \varepsilon
$$

Then the remaining assertions of Theorem 1.1 hold for the eigenspinor $\phi$.

1.2. Applications. Our hypothesis that $A_{d}$ and $A$ are Yang-Mills connections is stronger than necessary for many applications (indeed, we shall discuss one such application in $\$ 6$ (6): this is just the simplest way to state our main result. In practice, it is enough that (i) the curvatures $F_{A_{d}}$ and $F_{A}$ obey $C^{0}$ decay estimates of the shape (2.3) and (ii) that the conclusions of Lemma 5.5 hold with constant independent of $A_{d}$ or $A$. The latter condition is satisfied, for example, if the curvatures $F_{A_{d}}$ and $F_{A}$ obey $C^{l}$ decay estimates of the shape (2.4). A more detailed discussion of some important ways in which the hypotheses can be relaxed is given in $\S 2.2$ and $\$ 6$.

If $\phi \in L^{2}\left(\mathbb{R}^{4}, W \otimes E\right) \cap \operatorname{Ker} D_{A}$ then it is well-known that $|\phi|(x)=O\left(r^{-3}\right)$, for $r \rightarrow \infty$, so the estimate (1.5) for the rate of decay of $L^{2}$ harmonic spinors on $\mathbb{R}^{4}$ is sharp [9, Equation (3.2.24)]. (See [9, §3.3.3] for an explicit construction of solutions.) However, as we shall explain in $\$ 6$, the more interesting applications of Theorem 1.1 and Corollary 1.2 arise when $\phi$ is an $L^{2}$ eigenspinor over an annulus, with the given curvature constraints, rather than all of $\mathbb{R}^{4}$ or $S^{4}$. Moreover, while standard elliptic theory would predict an estimate with the general shape of (1.5), the crucial point is that we know the explicit dependence of the constant on the radii $r_{0}$ and $r_{1}$ and, indirectly via the bounds (1.2) and (1.3), on the curvatures of the connections defining the Dirac operator: as we sketch briefly below and in $\$ 6$, this is the significant feature of the estimate (1.5) which allows us to exploit it in gauge-theoretic applications such as the problem of gluing (and ungluing) PU(2) monopoles [13], [12], 15], [16].

A key difficulty in attempts to directly adapt Taubes' gluing arguments for anti-self-dual connections [31] to the case of $\mathrm{PU}(2)$ monopoles is the problem of obtaining useful $C^{0} \cap L_{2}^{2}$ estimates for negative spinors, namely sections $\phi$ of $C^{\infty}\left(W^{-} \otimes E\right)$; the Bochner formulas (4.4) imply that estimates for positive spinors, or sections of $W^{+} \otimes E$, are comparatively straightforward. If $\phi \in \operatorname{Ker} D_{A} \cap L^{2}\left(W^{-} \otimes E\right)$, however, the shape of the Bochner formulas (4.4) implies that elementary methods do not yield $C^{0} \cap L_{2}^{2}$ bounds on $\phi$ which are uniform with respect to $F_{A}$ if $A$ "bubbles" in the Uhlenbeck sense [32]. See $\delta[6$ for a more detailed explanation of the difficulty. While standard elliptic theory yields $C^{0}$ estimates for $\phi$ on the complement in $X$ of small balls $B\left(x_{i}, \delta_{i}\right)$, when $F_{A}$ is $C^{0}$ bounded on such a region (but has curvature which bubbles on the balls $\left.B\left(x_{i}, \delta_{i}\right)\right)$, these estimates will not necessarily be uniform with respect to the ball radii: this is a serious problem in gluing contexts, as one needs estimates which are uniform with respect to the radii $\delta_{i}$ as $\delta_{i} \rightarrow 0$. However, we see from Theorem 1.1 that the spinor $\phi$ is $C^{0}$ bounded on the complement of the ball $B\left(x_{0}, 2 r_{0}^{1 / 3}\right.$ ), with constant which is independent of $r_{0} \rightarrow 0$ (and also $r_{1} \rightarrow \infty$ ). Pointwise decay estimates with this uniformity property for the curvatures of Yang-Mills connections have been derived by Donaldson [8], Råde [27], and Groisser-Parker [21] and it is these decay estimates which motivate our hypotheses on the curvatures of the connections $\nabla_{g}$, $A_{d}$, and $A$; see $\delta 2.1$ for a fuller account. 
1.3. Main ideas in the proof. Theorem 1.1 can be proved in two quite different ways. The approach taken in the present article uses a pointwise Kato-Yau inequality,

$$
|\nabla| \phi||^{2} \leq\left(\frac{m-1}{m}\right)\left|\nabla_{A} \phi\right|^{2}
$$

which we show is obeyed by $D_{A}$-harmonic spinors over an $m$-dimensional manifold, coupled with the Bochner formula (4.4) for the Dirac Laplacian $D_{A}^{2}$. The Kato-Yau inequality also holds for an eigenspinor of $D_{A}$ with eigenvalue $\mu$ if $A$ is replaced by the Friedrich connection $\tilde{A}$ defined by $A$ and $\mu$ (see equation (3.4)). The inequality (1.8) then leads to a differential inequality for $|\phi|$ and hence a decay estimate. See [25] for a survey of such inequalities and applications [2], [23], [26], [29], [27], whose use was pioneered by Yau in his proof of the Calabi conjecture [35]. As pointed out to us by D. Yang [34], related differential inequalities for vector-valued harmonic functions on $\mathbb{R}_{+}^{n+1}$ were known to Stein [30, §VII.3.1].

After the preprint version of our article [11] was distributed, the preprints [5] and [6] became available. These articles describe Kato-Yau inequalities for injectively elliptic firstorder linear differential operators and compute the optimal Kato-Yau constants in a general setting; the recent article [7] contains a nice survey of these results due, independently, to Branson and to Calderbank, Gauduchon, and Herzlich.

A second (more cumbersome) proof of Theorem 1.1, described in a preliminary version of this article [11], considers the Dirac equation on the cylinder $\mathbb{R} \times S^{3}$ and hinges on a calculation of the first eigenvalue, $9 / 4$, of the square of the Dirac operator on $S^{3}$ for the standard metric [3], [19], 22].

Neither proof of Theorem 1.1 requires us to restrict our attention to dimension four. We restrict to the special case mainly because this is where our present applications lie and also for expository reasons, as the proof is easier to follow.

1.4. Outline of the article. We begin in $\$ 2$ by recalling the decay estimates we shall need for Yang-Mills connections (see $\$ 2.1$ ), as well as describing some natural situations where the hypotheses of Theorem 1.1 are known to hold (see $\$ 2.2$ ). In $\$ 3$ we show that harmonic spinors satisfy the pointwise Kato-Yau inequality (1.8). As we explain in $\S$, this leads to a useful differential inequality for $\Delta|\phi|^{2 / 3}$ (giving rise to the $O\left(r^{-3}\right)$ decay rate), rather than the weaker inequality for $\Delta|\phi|$ which is a consequence of the standard Kato estimate (and which would only lead to a $O\left(r^{-2}\right)$ decay rate and without the essential appearance of the constant $r_{0}$ in (1.5)). When integrated twice, this eventually leads to the decay estimate (1.5), as we show in $\$$, where we present our proof of Theorem 1.1. In $\$ 5.2$ we explain how Theorem 1.1 leads, almost immediately, to 1.2. Finally, in $\$ 6$ we describe an application of Theorem 1.1 to the problem of deriving uniform $L_{2}^{2} \cap C^{0}$ estimates for harmonic spinors when the connection $A$ may bubble.

Acknowledgments. I am grateful to Tom Mrowka for pointing out that calculations of the Dirac operator spectrum for $S^{3}$ are well-known and that such calculations can be found in [22]. I am also grateful to Hiraku Nakajima for directing me to the references [4] and [26] and to Deane Yang for describing the early use of Kato-Yau type differential inequalities in harmonic analysis [30, §VII.3.1]. Finally, I would like to thank the Institute for Advanced Study, Princeton, and the National Science Foundation, for their generous support during the preparation of this article. 


\section{Decay estimates for Yang-Mills connections And the hypotheses of THEOREM 1.1}

We review in $\$ 2.1$ the relevant decay estimates of Donaldson, Groisser-Parker and Råde in order to give some context to Theorem 1.1 and its corollaries, to explain the hypotheses on the curvature of the metric $g$ and curvatures $F_{A_{d}}$ and $F_{A}$, and to introduce some preliminary material we shall need for the remainder of the article. In $\$ 2.2$ we describe some situations of wider interest where the conditions on $G_{g}, \kappa_{g}, F_{A_{d}}$, and $F_{A}$ are known to hold.

2.1. Decay estimates for Yang-Mills connections. The simplest case, due to Donaldson and Råde, is when $X$ is $\mathbb{R}^{4}$ with its standard metric.

First, recall that the fundamental decay estimates, for a Yang-Mills connection $A$ over $B\left(0, r_{1}\right)-\{0\} \subset \mathbb{R}^{4}$ with $L^{2}$-small energy,

$$
\left|F_{A}\right|(x) \leq C\left([A], r_{1}\right) \frac{1}{r^{4}} \quad 0<r \leq r_{1} / 2,
$$

and, by a conformal diffeomorphism, for a Yang-Mills connection $A$ over $\mathbb{R}^{4}-B\left(0, r_{0}\right)$ with $L^{2}$-small energy,

$$
\left|F_{A}\right|(x) \leq C\left([A], r_{0}\right) \frac{1}{r^{4}} \quad 2 r_{0} \leq r<\infty,
$$

were first proved by Uhlenbeck [33, Corollary 4.2]. However, it is very useful in applications to gluing and ungluing anti-self-dual connections to have pointwise estimates of the above shape, but where (i) the constant $C$ at most depends on the connection $A$ through the $L^{2}$ norm of its curvature, $F_{A}$, (ii) the connection $A$ is only known to be Yang-Mills or anti-selfdual over an annulus $\Omega\left(r_{0}, r_{1}\right)$ with finite, positive inner and outer radii, rather than (as above) over a punctured ball, $B\left(0, r_{1}\right)-\{0\}$, or the complement of a ball, $\mathbb{R}^{4}-B\left(0, R_{1}\right)$, and (iii) the explicit dependence of $C$ on the radii $r_{0}, r_{1}$ is known. The earliest such refinement, for anti-self-dual connections, $A$, was due to Donaldson [8, Appendix], [9, Proposition 7.3.3] and extended by Råde [27, Theorem 1] to the case of Yang-Mills connections. The essential feature common to the estimate (1.5) above for harmonic spinors and (2.3), (2.6), and (2.8) below for Yang-Mills connections, is that the constants on the right (i) at most depend on the $L^{2}$ norm of $F_{A}$ and (ii) the precise dependence on the radii $r_{0}, r_{1}$ is known.

Theorem 2.1. [9, Proposition 7.3.3], [27, Theorem 1] There exist positive constants $c$, $\varepsilon$ with the following significance. If $0<4 r_{0}<r_{1}<\infty, A$ is a Yang-Mills connection on $\Omega\left(r_{0}, r_{1}\right) \subset \mathbb{R}^{4}$, with its Euclidean metric, with $\left\|F_{A}\right\|_{L^{2}\left(\Omega\left(r_{0}, r_{1}\right)\right)} \leq \varepsilon$, and $r=|x|$, then for $x \in \Omega\left(2 r_{0}, r_{1} / 2\right)$,

$$
\left|F_{A}\right|(x) \leq c\left(r_{0}^{2} r^{-4}+r_{1}^{-2}\right)\left\|F_{A}\right\|_{L^{2}\left(\Omega\left(r_{0}, r_{1}\right)\right)} .
$$

Though not mentioned explicitly in [9], 27], the proof of Theorem 2.1 extends to give the following more general decay estimate:

Corollary 2.2. Continue the hypotheses of Theorem 2.1. Then for any integer $k \geq 0$ we have

$$
\left|\nabla_{A}^{k} F_{A}\right|(x) \leq c_{k} r^{-k}\left(r_{0}^{2} r^{-4}+r_{1}^{-2}\right)\left\|F_{A}\right\|_{L^{2}\left(\Omega\left(r_{0}, r_{1}\right)\right)} .
$$

For example, the corollary follows immediately by combining Råde's Lemma 2.2 and Theorem $1^{\prime}$ (the version of his Theorem 1 for a cylinder $\left(t_{0}, t_{1}\right) \times S^{3}$ in place of the annulus $\left.\Omega\left(r_{0}, r_{1}\right)\right)$. 
There are a couple of standard, useful situations, which we now discuss, where a metric $g$ approximates a Euclidean metric well enough that analogues of Theorem 2.1 hold on such Riemannian manifolds, though some work is involved in order to adapt Råde's argument to these more general cases. The relevant extensions are due to Groisser and Parker.

First, suppose that $(X, g)$ is an asymptotically flat four-manifold with a single end. Thus, $X$ is a disjoint union $X_{0} \cup X_{\infty}$, where $X_{0}$ is compact and for some $0<R<\infty$ there is a diffeomorphism of $X_{\infty} \cong \mathbb{R}^{4}-B(0, R)$, giving coordinates $\left\{y^{i}\right\}$ on $X_{\infty}$ with respect to which the metric $g$ has the form

where, denoting $r=|y|$,

$$
g_{i j}=\delta_{i j}+h_{i j}
$$

$$
r^{2}\left|h_{i j}\right|+r^{3}\left|\partial_{\alpha} h_{i j}\right|+r^{4}\left|\partial_{\alpha} \partial_{\beta} h_{i j}\right| \leq c(g)
$$

for some positive constant $c(g)$. Let $\Omega\left(r_{0}, r_{1}\right)$ denote the annulus $r_{0}<r<r_{1}$ in $\mathbb{R}^{4}-$ $B(0, R) \cong X_{\infty}$.

Theorem 2.3. 21, Theorem 1.2] Let $(X, g)$ be an asymptotically flat four-manifold and let $E$ be a Hermitian vector bundle over $X$. Then there exist positive constants $c, \varepsilon, R$ such that if $4 R \leq 4 r_{0} \leq r_{1}<\infty$ and $A$ is a Yang-Mills connection on $\Omega\left(r_{0}, r_{1}\right)$ with $\left\|F_{A}\right\|_{L^{2}\left(\Omega\left(r_{0}, r_{1}\right)\right)} \leq \varepsilon$, and $r=|y|$, then for $y \in \Omega\left(2 r_{0}, r_{1} / 2\right)$,

$$
\left|F_{A}\right|_{g}(y) \leq c\left(r_{0}^{2} r^{-4}+r_{1}^{-2}\right)\left\|F_{A}\right\|_{L^{2}\left(\Omega\left(r_{0}, r_{1}\right)\right)} .
$$

Though not explicitly proved in [21], the proof of Corollary 2.2 also yields more general decay estimates in the situation of Theorem 2.3.

$$
\left|\nabla_{A}^{k} F_{A}\right|_{g}(y) \leq c_{k} r^{-k}\left(r_{0}^{2} r^{-4}+r_{1}^{-2}\right)\left\|F_{A}\right\|_{L^{2}\left(\Omega\left(r_{0}, r_{1}\right)\right)}
$$

Second, suppose $(X, g)$ is four-manifold of bounded geometry, namely positive injectivity radius and Riemannian curvature bounded in $C^{2}$. In 21] Groisser and Parker first prove Theorem 2.3 and then deduce Theorem 2.4 below as a corollary using a conformal diffeomorphism from $B(0, \rho)-\{0\}$, with metric $g$ on $B(0, \rho)$ of bounded geometry, onto $\mathbb{R}^{4}-B\left(0, \rho^{-1}\right)$, endowed with an induced metric $g_{\infty}$ which is observed to be asymptotically flat.

Theorem 2.4. [21, Theorem 1.1] Let $(X, g)$ be a Riemannian, smooth four-manifold of bounded geometry. Let $E$ be a Hermitian vector bundle over $X$. Then there exist positive constants $c, \varepsilon, \rho$ such that if $0<4 r_{0}<r_{1} \leq \rho$ and $A$ is a Yang-Mills connection on $\Omega\left(x_{0}, r_{0}, r_{1}\right)$ with $\left\|F_{A}\right\|_{L^{2}\left(\Omega\left(x_{0}, r_{0}, r_{1}\right)\right)} \leq \varepsilon$, and $r=\operatorname{dist}_{g}\left(x, x_{0}\right)$, then for $x \in \Omega\left(x_{0}, 2 r_{0}, r_{1} / 2\right)$,

$$
\left|F_{A}\right|_{g}(x) \leq c\left(r_{0}^{2} r^{-4}+r_{1}^{-2}\right)\left\|F_{A}\right\|_{L^{2}\left(\Omega\left(x_{0}, r_{0}, r_{1}\right)\right)} .
$$

Again, in the situation of Theorem 2.4, one has stronger decay estimates:

$$
\left|\nabla_{A}^{k} F_{A}\right|_{g}(x) \leq c_{k} r^{-k}\left(r_{0}^{2} r^{-4}+r_{1}^{-2}\right)\left\|F_{A}\right\|_{L^{2}\left(\Omega\left(x_{0}, r_{0}, r_{1}\right)\right)} .
$$

2.2. Some applications where the hypotheses of Theorem 1.1 hold. We begin with the constraints on the Riemannian geometry of $(X, g)$. First, the hypotheses (1.1) and (1.2) obviously hold when $(X, g)$ is $\mathbb{R}^{4}$ with its Euclidean metric. Second, suppose $(X, g)$ is a Riemannian, smooth four-manifold of bounded geometry. Let $B\left(x_{0}, \rho\right) \subset X$ be a geodesic ball and suppose that $\rho$ is much less than the injectivity radius of $(X, g)$. Then Lemma 5.3 implies that the Green kernel $G_{g}$ obeys (1.1). The scalar curvature $\kappa_{g}$ obeys (1.2) since, for $x \in B\left(x_{0}, \rho\right)-\left\{x_{0}\right\}$ and $r=\operatorname{dist}_{g}\left(x, x_{0}\right)$,

$$
\left|\kappa_{g}\right|(x) \leq c_{1} \varepsilon r^{-2} \quad \text { and } \quad\left|\kappa_{g}\right|(x) \leq c_{2} r_{1}^{-2} \leq c_{2}\left(r_{0}^{2} r^{-4}+r_{1}^{-2}\right) \text {, }
$$


where we choose $c_{1}=\left\|\kappa_{g}\right\|_{L^{\infty}\left(B\left(x_{0}, \rho\right)\right)}, \varepsilon=\rho^{2} \ll 1$, and $c_{2}=\rho^{2}\left\|\kappa_{g}\right\|_{L^{\infty}\left(B\left(x_{0}, \rho\right)\right)}$, with $r_{1} \leq \rho$. Third, suppose $(X, g)$ is an asymptotically flat four-manifold. Then Lemma 5.2 now implies that the Green kernel $G_{g}$ obeys (1.1) while our definition (2.5) of an asymptotically flat Riemannian four-manifold ensures that the scalar curvature $\kappa_{g}$ obeys (1.2).

We now turn to the hypotheses on the curvatures $F_{A_{d}}$ and $F_{A}$. First, it is not necessary that $A_{d}$ and $A$ be Yang-Mills connections, but rather that their curvatures satisfy the conditions (i) and (ii) described at the beginning of $\$ 1.2$. Second, the connections $A$ of interest in [16], [17 are the connection components of "approximate $\mathrm{PU}(2)$ monopoles" obtained by splicing anti-self-dual connections $A_{i}$ over $S^{4}$ onto background connections $A_{0}$ varying in an Uhlenbeck-compact family. Thus, over small balls $B\left(x_{i}, 4 \sqrt{\lambda_{i}}\right)$ in $X$, the connection $A$ obeys the decay estimate (2.4) because the connections $A_{i}$ are anti-self-dual. On the other hand, over the complement of these balls, the background connection $A_{0}$ over $X$ obeys a decay estimate of the shape (2.4) (with $\left\|F_{A_{0}}\right\|_{L^{2}\left(\Omega\left(r_{0}, r_{1}\right)\right)}$ replaced by $\varepsilon$ ) because, for $x \in B\left(x_{0}, \rho\right)-\left\{x_{0}\right\}$, we may write

$$
\left|\nabla_{A_{0}}^{k} F_{A_{0}}\right|_{g}(x) \leq c_{k}^{\prime} \varepsilon r^{-k} r_{1}^{-2} \leq c_{k}^{\prime} \varepsilon r^{-k}\left(r_{0}^{2} r^{-4}+r_{1}^{-2}\right),
$$

where $c_{k}^{\prime}=\left\|\nabla_{A_{0}}^{k} F_{A_{0}}\right\|_{L^{\infty}\left(B\left(x_{0}, \rho\right)\right)}$ and $\varepsilon=\rho^{k+2} \ll 1$ with $r_{1} \leq \rho$. In applications such as those of [16], when $E$ has complex rank two, if the connection $A$ on $E$ corresponds to a unitary connection $A_{e}$ on $\operatorname{det}(E)$ and an orthogonal connection $\hat{A}$ on $\mathfrak{s u}(E)$, there is no loss in generality if one assumes that the determinant connections $A_{d}$ and $A_{e}$ are Yang-Mills, and so Theorems 2.1, 2.3, or 2.4, together with their easy corollaries, guarantee that the constraints on $A_{e}$ and $A_{d}$ are satisfied. Thus one need only ensure that $\hat{A}$ also obeys the required estimates.

\section{A Kato-Yau inequality for eigenspinors}

Recall that any smooth section $\phi$ of a Riemannian vector bundle with orthogonal connection $A$ satisfies the pointwise Kato inequality [18, Equation (6.20)]:

$$
|\nabla| \phi|| \leq\left|\nabla_{A} \phi\right| \text {. }
$$

If $\phi$ is not arbitrary, but rather satisfies a differential equation, then the preceding inequality can sometimes be improved to

$$
(1+\delta)|\nabla| \phi|| \leq\left|\nabla_{A} \phi\right|
$$

for some positive constant $\delta$. For example, if $\phi=F_{A} \in C^{\infty}\left(\Lambda^{2} \otimes \mathfrak{u}(E)\right)$ is the curvature of a Yang-Mills connection $A$ on a Hermitian vector bundle $E$ over a Riemannian four-manifold $X$, then $F_{A}$ satisfies the Kato-Yau inequality

$$
\left.|\nabla| F_{A}\right|^{2} \leq \frac{2}{3}\left|\nabla_{A} F_{A}\right|^{2}
$$

See [27, Lemma 3.1] for Råde's proof when $A$ is Yang-Mills and [23, §4.2] for Itoh-Nakajima's argument when $A$ is (anti-)self-dual. A related result is proved by Bando-Kasue-Nakajima in [2, Lemma 4.9], though they attribute the trick to Yau [4], 25], [26], [29], [35].

We shall derive a similar Kato-Yau inequality for harmonic spinors. Given a Riemannian $m$-manifold $(X, g)$, let $V$ be a complex Hermitian vector bundle over $X$ with a unitary connection $\nabla_{A}$, and let $\rho: T^{*} X \rightarrow \operatorname{End}_{\mathbb{C}}(V)$ be a linear map such that for all $\alpha \in \Omega^{1}(X)$,

- $\rho(\alpha)^{\dagger}=-\rho(\alpha)$ and $\rho(\alpha)^{2}=-g(\alpha, \alpha)$,

- $\left[\nabla_{A}, \rho(\alpha)\right]=\rho(\nabla \alpha)$, where $\nabla$ is the Levi-Civita connection on $T^{*} X$. 
The composition $D_{A}=\rho \circ \nabla_{A}$ of Clifford multiplication $\rho: T^{*} X \rightarrow \operatorname{End}_{\mathbb{C}}(V)$ and the covariant derivative $\nabla_{A}: C^{\infty}(V) \rightarrow C^{\infty}\left(T^{*} X \otimes V\right)$ is a generalized Dirac operator and $(\rho, V)$ is a (complex) Dirac bundle in the sense of [24, Definition II.5.2]. Now suppose that $\phi \in C^{\infty}(V)$ is $D_{A \text {-harmonic: }}$

$$
D_{A} \phi=\sum_{i=1}^{m} \rho\left(e^{i}\right) \nabla_{A, e_{i}} \phi=0 .
$$

Recall that for $f \in C^{\infty}(X)$, we have $\nabla f=\langle\cdot, \operatorname{grad} f\rangle$, so $|\nabla f|=|\operatorname{grad} f|$ and $\nabla_{e} f=$ $\langle e, \operatorname{grad} f\rangle$ for $e \in C^{\infty}(T X)$. If we choose $e=(|\operatorname{grad} f|)^{-1} \operatorname{grad} f$ at points where $(\nabla f)(x) \neq$ 0 then we obtain the familiar identity

$$
\left|\nabla_{e} f\right|=|\nabla f| .
$$

We may suppose without loss of generality that $x \in X$ is a point for which $|\phi|(x) \neq 0$ and $\nabla|\phi|(x) \neq 0$. At any such point $x$ we can find an orthonormal frame $\left\{e_{i}\right\}$ for $T X$ and dual coframe $\left\{e^{i}\right\}$ for $T^{*} X$ such that

$$
|\nabla| \phi||=\left|\nabla_{e_{1}}\right| \phi|| \leq\left|\nabla_{A, e_{1}} \phi\right| .
$$

Indeed, we can take $e_{1}=(|\operatorname{grad}| \phi||)^{-1} \operatorname{grad}|\phi|$ and complete this to give a local orthonormal frame for $T X$ near $x$. Note that $\left|\rho\left(e^{\prime}\right) \phi\right|=|\phi|$ for any $e^{\prime} \in C^{\infty}\left(T^{*} X\right)$ with $\left|e^{\prime}\right|=1$, so

$$
\begin{aligned}
\left|\nabla_{A, e_{1}} \phi\right|^{2} & =\left|\rho\left(e^{1}\right) \nabla_{A, e_{1}} \phi\right|^{2} \leq\left|\sum_{i=2}^{m} \rho\left(e^{i}\right) \nabla_{A, e_{i}} \phi\right|^{2} \\
& \leq\left(\sum_{i=2}^{m}\left|\nabla_{A, e_{i}} \phi\right|\right)^{2} \leq(m-1) \sum_{i=2}^{m}\left|\nabla_{A, e_{i}} \phi\right|^{2},
\end{aligned}
$$

using $\left(\sum_{i=1}^{n} a_{i}\right)^{2} \leq n \sum_{i=1}^{n} a_{i}^{2}$ to obtain the final inequality. Thus,

$$
m\left|\nabla_{A, e_{1}} \phi\right|^{2} \leq(m-1) \sum_{i=1}^{m}\left|\nabla_{A, e_{i}} \phi\right|^{2}=(m-1)\left|\nabla_{A} \phi\right|^{2} .
$$

Combining inequalities (3.2) and (3.3) yields the following Kato-Yau type inequality on the open set where $\phi(x) \neq 0$ and $\nabla|\phi|(x) \neq 0$. The inequality trivially holds where $\nabla|\phi|(x)=0$.

Lemma 3.1. Let $(\rho, V)$ be a spin ${ }^{c}$ structure over a Riemannian m-manifold $(X, g)$ with spin connection $\nabla_{A}$ on $V$. Then, for any smooth section $\phi \in C^{\infty}(X, V)$ for which $D_{A} \phi=0$, the following pointwise inequality holds on the open subset $\{\phi \neq 0\} \subset X$ :

$$
|\nabla| \phi||^{2} \leq\left(\frac{m-1}{m}\right)\left|\nabla_{A} \phi\right|^{2} \quad \text { on } X \text {. }
$$

One can extend the preceding inequality to the case of eigenspinors with non-zero eigenvalue (see, for example, the proof of Theorem 4 in $[\mathbb{6}]$ ). Suppose $D_{A} \phi=\mu \phi$ for some $\mu \in \mathbb{R}$. The (metric) Friedrich connection [19] associated to $\nabla_{A}$ and $\mu$ is defined by

$$
\nabla_{\tilde{A}, \eta}=\nabla_{A, \eta}+\frac{\mu}{m} \rho(g(\cdot, \eta)), \quad \eta \in C^{\infty}(T X) .
$$

Then $\phi$ is harmonic with respect to the Dirac operator $D_{\tilde{A}}=\rho \circ \nabla_{\tilde{A}}=D_{A}-\mu$ and so one obtains a Kato-Yau inequality for eigenspinors: 
Lemma 3.2. Continue the hypotheses of Lemma 3.1, but suppose more generally that $\left(D_{A}-\mu\right) \phi=0$ for some $\mu \in \mathbb{R}$ and let $\tilde{A}$ be the Friedrich connection associated with $(A, \mu)$. Then, the following pointwise inequality holds on the open subset $\{\phi \neq 0\} \subset X$ :

$$
|\nabla| \phi||^{2} \leq\left(\frac{m-1}{m}\right)\left|\nabla_{\tilde{A}} \phi\right|^{2} \quad \text { on } X
$$

\section{Differential inequalities For Eigenspinors}

Using our Kato-Yau inequality we derive the differential inequalities satisfied by suitable powers of pointwise norms of eigenspinors.

4.1. Differential inequalities implied by the standard Kato estimate. Let $(\rho, W)$ be a $\operatorname{spin}^{c}$ structure on the Riemannian $m$-manifold $(X, g)$ with spin connection on a complex Hermitian bundle $W$ of rank $2^{n}$, where $m=2 n$ or $2 n+1$, let $E$ be a complex Hermitian bundle over $X$ equipped with a unitary connection $A$, and let $V=W \otimes E$.

If $m$ is even (respectively, odd), let $A_{d}$ denote the fixed unitary connection on $\operatorname{det}\left(W^{+}\right)$ (respectively, $\operatorname{det}(W)$ ), where $W=W^{+} \oplus W^{-}$, and give $W$ the spin connection induced by $A_{d}$ and the Levi-Civita connection on $T^{*} X$. To appreciate the significance of Lemma 3.1, note that for any $\phi \in C^{\infty}(V)$ the standard identity

$$
\Delta|\phi|^{2}=2\left\langle\nabla_{A}^{*} \nabla_{A} \phi, \phi\right\rangle-2\left|\nabla_{A} \phi\right|^{2}
$$

and the usual pointwise Kato inequality [18, Equation (6.20)],

$$
|\nabla| \phi|| \leq\left|\nabla_{A} \phi\right|,
$$

yields the differential inequality [18, Equation (6.21)] on the subset $\{\phi \neq 0\} \subset X$ :

$$
\Delta|\phi| \leq|\phi|^{-1}\left\langle\nabla_{A}^{*} \nabla_{A} \phi, \phi\right\rangle .
$$

Now the Bochner formula for $\nabla_{A}^{*} \nabla_{A}$ on $C^{\infty}(V)$ takes the general form [12, Lemma 4.1]

$$
D_{A}^{2} \phi=\nabla_{A}^{*} \nabla_{A} \phi+\frac{\kappa_{g}}{4} \phi+\rho\left(F_{A}\right) \phi+\frac{1}{2} \rho\left(F_{A_{d}}\right) \phi
$$

where $\kappa_{g}$ is the scalar curvature of the Levi-Civita connection on $T^{*} X$. (When restricted to $C^{\infty}\left(V^{ \pm}\right)$and $X$ has dimension four, the curvature terms $F_{A}$ and $F_{A_{d}}$ above can be replaced by $F_{A}^{ \pm}$and $F_{A_{d}}^{ \pm}$: this leads to useful, global pointwise estimates for sections of $V^{+}$ but not for $V^{-}$, as $F_{A}^{-}$will not be uniformly $L^{p}$ bounded with respect to $A$ when $p>2$ in applications of interest [16].) When $D_{A} \phi=0$, the inequality (4.3) and the identity (4.4) imply

$$
\Delta|\phi| \leq c\left(\left|\kappa_{g}\right|+\left|F_{A}\right|+\left|F_{A_{d}}\right|\right)|\phi| .
$$

We would therefore only expect the standard Kato inequality to at most imply an $r^{-2}$ decay estimate for $|\phi|(x)$ on $\mathbb{R}^{4}$ with its standard metric, as we can see from our proof of Theorem 1.1, while our Kato-Yau inequality (Lemma 3.1) implies a $r^{-3}$ decay estimate for $|\phi|(x)$ as we explain in the next subsection. 
4.2. Differential inequalities implied by the Kato-Yau estimate. The $r^{-4}$ decay estimates of Groisser-Parker, Råde, and Uhlenbeck hinge on differential inequalities for $\Delta\left|F_{A}\right|^{1 / 2}$ rather than $\Delta\left|F_{A}\right|$. To obtain differential inequality in our case which yields a decay rate strictly faster than $r^{-2}$, suppose $0<\alpha<1$ and observe that

$$
\begin{aligned}
\Delta|\phi|^{\alpha} & =\alpha|\phi|^{\alpha-2}\left((1-\alpha)(\nabla|\phi|)^{2}+|\phi| \Delta|\phi|\right) \\
& =\alpha|\phi|^{\alpha-2}\left((2-\alpha)(\nabla|\phi|)^{2}+\frac{1}{2} \Delta|\phi|^{2}\right) .
\end{aligned}
$$

(Our convention, when $X=\mathbb{R}^{4}$ with its standard metric, gives $\Delta=-\sum_{i=1}^{4} \partial^{2} / \partial x_{i}^{2}$, which has sign opposite to that used in [20].) Combining this with identity (4.1) implies that

$$
\Delta|\phi|^{\alpha}=\alpha|\phi|^{\alpha-2}\left((2-\alpha)(\nabla|\phi|)^{2}+\left\langle\nabla_{A}^{*} \nabla_{A} \phi, \phi\right\rangle-\left|\nabla_{A} \phi\right|^{2}\right) .
$$

Thus, we see that the standard Kato inequality does not lead to a useful differential inequality for $\Delta|\phi|^{\alpha}$ when $\alpha<1$, but when $D_{A} \phi=0$, as we now assume, the refinement in Lemma 3.1 gives $|\nabla| \phi||^{2} \leq \frac{3}{4}\left|\nabla_{A} \phi\right|^{2}$ when $m=4$ and

$$
\begin{aligned}
\Delta|\phi|^{\alpha} & \leq \alpha|\phi|^{\alpha-2}\left((2-\alpha) \frac{3}{4}\left|\nabla_{A} \phi\right|^{2}+\left\langle\nabla_{A}^{*} \nabla_{A} \phi, \phi\right\rangle-\left|\nabla_{A} \phi\right|^{2}\right) \\
& =\alpha|\phi|^{\alpha-2}\left(((2 / 3)-\alpha) \frac{3}{4}\left|\nabla_{A} \phi\right|^{2}+\left\langle\nabla_{A}^{*} \nabla_{A} \phi, \phi\right\rangle\right) .
\end{aligned}
$$

Therefore, choosing $\alpha=2 / 3$, we obtain

$$
\Delta|\phi|^{\alpha} \leq \alpha|\phi|^{\alpha-2}\left\langle\nabla_{A}^{*} \nabla_{A} \phi, \phi\right\rangle .
$$

We now combine the preceding inequality with the Bochner identity (4.4) to give

$$
\Delta|\phi|^{2 / 3} \leq c\left(\left|\kappa_{g}\right|+\left|F_{A}\right|+\left|F_{A_{d}}\right|\right)|\phi|^{2 / 3} \quad \text { on } X .
$$

This inequality yields the estimate (4.8) when $\mu=0$. If $D_{A} \phi=\mu \phi$, for some $\mu \in \mathbb{R}$, then estimate (4.6) holds with the connection $A$ replaced by the Friedrich connection (3.4):

$$
\tilde{A}=A+\frac{\mu}{4} \sum_{i=1}^{4} \rho\left(e^{i}\right) e^{i} .
$$

Thus modified, inequality (4.6) yields the estimate (4.8) when $\mu \neq 0$ by writing $F_{\tilde{A}}$ in terms of $F_{A}$ and $\mu$. We have proved:

Lemma 4.1. Let $(\rho, W)$ be a spinc structure over a Riemannian four-manifold $(X, g)$ with unitary connection $A_{d}$ on $\operatorname{det}\left(W^{+}\right)$and spin connection on the Hermitian rank-4 bundle $W=W^{+} \oplus W^{-}$induced by $A_{d}$ and the Levi-Civita connection on $T^{*} X$. Let $E$ be a Hermitian bundle over $X$, endowed with a unitary connection $A$, let $V^{ \pm}=W^{ \pm} \otimes E$, and let $V=V^{+} \oplus V^{-}$. Let $\kappa_{g}$ be the scalar curvature of the Levi-Civita connection. If $\phi \in C^{\infty}(X, V)$ satisfies $D_{A} \phi=\mu \phi$ on $X$, for some $\mu \in \mathbb{R}$, then

$$
\Delta|\phi|^{2 / 3} \leq c\left(\mu^{2}+\left|\kappa_{g}\right|+\left|F_{A}\right|+\left|F_{A_{d}}\right|\right)|\phi|^{2 / 3} \quad \text { on } X .
$$

In applications such as those discussed in [12], [15], [13], the choice of unitary connection $A_{d}$ on $\operatorname{det}\left(W^{+}\right)$is arbitrary. Typically, therefore, we would assume without loss of generality that $A_{d}$ is Yang-Mills and so $F_{A_{d}}$ satisfies the same decay estimates as $F_{A}$. For the sake of exposition in the remainder of the article, as it does not affect the proof, we shall assume $c_{1}\left(W^{+}\right)=0$ and that $A_{d}$ is flat, so $F_{A_{d}}=0$ in inequalities such as (4.8) above. 
As we shall shortly see, the Kato-Yau estimate (and the differential inequality (4.5) which it implies) is the key ingredient which leads to the stronger $r^{-3}$ estimate of Theorem 1.1.

As pointed out to us by D. Yang [34], arguments of the kind described above leading to useful differential inequalities for $\Delta|u|^{\alpha}$ have been known for some time in harmonic analysis, where $u \in C^{\infty}\left(\Omega, \mathbb{R}^{n+1} \otimes \mathbb{R}^{N}\right)$ is a solution to the generalized Cauchy-Riemann equations on an open subset $\Omega$ of the half-space $\mathbb{R}_{+}^{n+1}[30$, §VII.3.1].

\section{Decay estimates for $L^{2}$ Eigenspinors on Riemannian manifolds}

In this section we prove Theorem 1.1 and Corollary 1.2, using our differential inequality for eigenspinors (Lemma 4.1). While our argument superficially follows the broad pattern of the proofs of [27, Theorem 1] and [21, Theorems 1.1 \& 1.2], the main new difficulty lies in finding suitable comparison functions for our applications of the maximum principle, which differ in subtle ways from those of [21] and 27], as well as a realization of the correct decay conditions one needs to impose on the curvatures $F_{A}$ and $\kappa_{g}$.

5.1. Decay estimates for harmonic spinors and proof of Theorem 1.1. We first deal with the case when the spinor is harmonic. We begin by recalling an elementary comparison lemma:

Lemma 5.1. 27, Lemma 3.2], [21, Lemma 3.2] Let $h$ be a positive harmonic function on a domain $\Omega$ in a Riemannian manifold. Set $\xi=|d(\log \sqrt{h})|^{2}$. Then for any $a \geq-1$ the operator $L=\Delta+a \xi$ satisfies the comparison principle on $\Omega$. Specifically, if $u, w \in L_{1}^{2}(\Omega)$ with $u \leq w$ weakly on $\partial \Omega$ and $L u \leq L w$ weakly on $\Omega$, then $u \leq w$ a.e. on $\Omega$.

As Råde points out in [27], an interesting feature of Lemma 5.1 is that we are allowed to choose $a<0$ and so have $a \xi<0$ on $\Omega$. However, while the comparison theorem holds for $\Delta+c$, where $c \geq 0$ is any function, it does not hold in general for $c<0$ on $\Omega$ (for example, a negative constant) (see [20, Theorem 3.3] and the remarks preceding its statement, noting that their sign conventions for the Laplacian are opposite to ours).

To apply Lemma 5.1 and ensure that the hypothesis (1.1) on the Green kernel of $(X, g)$ is obeyed in the three geometric situations discussed in $\$ 2.2$, we need a positive harmonic function $h$ where both $h$ and $\xi=|d(\log \sqrt{h})|^{2}$ are essentially $1 / r^{2}$ near the point $x_{0}$. The case $X=\mathbb{R}^{4}$ with its Euclidean metric is trivial, so we consider the remaining two situations of interest to us. The first and more subtle construction, where $(X, g)$ is asymptotically flat, is due to Groisser and Parker:

Lemma 5.2. 21, Proposition 3.3] Let $(X, g)$ be an asymptotically flat Riemannian fourmanifold. Then there is a positive constant $\rho_{0}$ such that for each $\rho<\rho_{0}$ there is a positive harmonic function $h$ on $X-B\left(x_{0}, \rho^{-1}\right)$ with $h=\rho^{2}$ on $\partial B\left(x_{0}, \rho^{-1}\right)$ and, if $r=\operatorname{dist}_{g}\left(x, x_{0}\right) \geq$ $\rho^{-1}$,

$$
\frac{3}{4 r^{2}} \leq h \leq \frac{4}{3 r^{2}} \quad \text { and } \quad h \leq 8 \xi
$$

Second, we consider a small ball in a Riemannian four-manifold $(X, g)$ :

Lemma 5.3. Let $(X, g)$ be a Riemannian four-manifold of bounded geometry. Then there is a positive constant $\rho_{0}$ such that for each $\rho<\rho_{0}$ there is a positive harmonic function $h$ on $B\left(x_{0}, \rho\right)$ with $h=1 / \rho^{2}$ on $\partial B\left(x_{0}, \rho\right)-\left\{x_{0}\right\}$ and, if $r=\operatorname{dist}_{g}\left(x, x_{0}\right) \leq \rho$,

$$
\frac{3}{4 r^{2}} \leq h \leq \frac{4}{3 r^{2}} \quad \text { and } \quad h \leq 8 \xi
$$


Proof. First, on $\mathbb{R}^{4}$ with its standard metric, the Green's function for the Laplacian on functions is $G(x, y)=-\left(8 \pi^{2}\right)^{-1}|x-y|^{-2}$, so we can choose

$$
h(x)=\frac{1}{|x|^{2}}=-8 \pi^{2} G(x, 0), \quad x \in \mathbb{R}^{4}-\{0\} .
$$

Then $h=\xi$ and so satisfies the constraints in this case. In general, note that $g_{i j}=$ $\delta_{i j}+O\left(r^{2}\right), \partial_{k} g_{i j}=O(r)$, and $\partial_{k} \partial_{l} g_{i j}=O(1)$. The existence of $h$ on $B\left(x_{0}, \rho\right)-\left\{x_{0}\right\}$ now follows from its existence when $g$ is flat and the construction of the Green kernel in [1, §4.2] for arbitrary metrics.

Remark 5.4. The values of the constants $c=3 / 4, c^{-1}=4 / 3$ in inequalities (5.2) are not critical. Though any $c \in(0,1)$ would suffice, the construction of suitable sub- and sup-solutions is a little easier to follow by the provision of specific constants.

For the remainder of this section we shall assume, for the sake of exposition, that we are in the situation of Lemma 5.3; there is no essential difference in the proof for the asymptotically flat case. Now fix $4 r_{0}<r_{1} \leq \rho_{0}$ where $\rho_{0}$ is small enough that Lemma 5.3 applies. Set $r(x)=\operatorname{dist}_{g}\left(x, x_{0}\right)$ on $X$.

In our applications of Lemma 5.1 we shall need an estimate for $|\phi|$ on the boundaries of the annulus $\Omega\left(x_{0}, 2 r_{0}, r_{1} / 2\right)$, corresponding to the boundary estimates for $\left|F_{A}\right|$ in equation (3.9) in [21], where such estimates follow from [21, Equation (3.4)].

Lemma 5.5. Continue the hypotheses of Theorem 1.1. Then for any integer $k \geq 0$, there is a constant $c_{k}$ (independent of the connections $A_{d}$ on $\operatorname{det}\left(W^{+}\right)$and $A$ on $E$ ) such that

$$
\left|\nabla_{A}^{k} \phi\right|_{g}(x) \leq c_{k} r^{-k-2}\|\phi\|_{L^{2}\left(\Omega_{g}\left(x_{0}, r / 2,2 r\right), g\right)} \quad \text { on } \Omega_{g}\left(x_{0}, 2 r_{0}, r_{1} / 2\right) \text {. }
$$

Proof. Let $r \in\left[2 r_{0}, r_{1} / 2\right]$ be a constant and let $\tilde{g}=r^{-2} g$, so that $\Omega_{g}(r / 2,2 r)=\Omega_{\tilde{g}}(1 / 2,2)$. Then $D_{A}^{\tilde{g}} \phi=r D_{A}^{g} \phi=0$ and so elliptic estimates on the rescaled annulus $\Omega_{\tilde{g}}(1 / 2,2)$ and the Sobolev embedding $L_{3}^{2} \subset C^{0}$ give

$$
\begin{aligned}
r^{k}\left|\nabla_{A}^{k} \phi\right|_{g}(x) & =\left|\nabla_{A}^{k} \phi\right|_{\tilde{g}}(x) \\
& \leq c\|\phi\|_{L_{k+3, A}^{2}\left(\Omega_{\tilde{g}}(3 / 4,4 / 3)\right)} \quad\left(\text { on } \Omega_{\tilde{g}}(3 / 4,4 / 3)\right) \\
& \leq c_{k}\|\phi\|_{L^{2}\left(\Omega_{\tilde{g}}(1 / 2,2)\right)} \\
& =c_{k} r^{-2}\|\phi\|_{L^{2}\left(\Omega_{g}(r / 2,2 r)\right)},
\end{aligned}
$$

and the result follows. The hypotheses of Theorem 1.1 on the connection $A$ ensure that the Sobolev and elliptic estimates above are uniform with respect to $A$.

At this point we shall employ the scaling argument in the proof of Lemma 5.5 to make a further simplification to the proof of Theorem 1.1.

Lemma 5.6. If the estimate (1.5) is valid for some fixed constant $\tilde{r}_{0}>0$ and any $\tilde{r}_{1}>4 \tilde{r}_{0}$, then it holds for any pair of rescaled constants $\left(r_{0}, r_{1}\right)=\lambda\left(\tilde{r}_{0}, \tilde{r}_{1}\right), \lambda>0$, for which the hypotheses of Theorem 1.1 are satisfied.

Proof. First observe that the conditions (1.1), (1.2), (1.3), and (1.4) are scale-invariant, as can be seen by replacing the metric $g$ by a rescaled metric $\tilde{g}=\lambda^{-2} g$ for any $\lambda>0$, and using the rescaling argument of the next paragraph. Hence, the constants $c_{0}, c_{1}$, and $c_{2}$ are also invariant under rescaling. 
Let $0<4 r_{0}<r_{1}<\infty$ and define $\lambda>0$ by requiring that $r_{0}=\lambda \tilde{r}_{0}$. By hypothesis, Theorem 1.1 holds for the metric $\tilde{g}=\lambda^{-2} g$ and radii $\tilde{r}_{0}=r_{0} / \lambda, \tilde{r}_{1}=r_{1} / \lambda>4 \tilde{r}_{0}$, and $\tilde{r}=r / \lambda \in\left[2 \tilde{r}_{0}, \tilde{r}_{1} / 2\right]$, where $r=\operatorname{dist}_{g}\left(x, x_{0}\right)$ and $\tilde{r}=\operatorname{dist}_{\tilde{g}}\left(x, x_{0}\right)$. Thus, for $2 \tilde{r}_{0} \leq \tilde{r} \leq \tilde{r}_{1} / 2$,

$$
\left|\nabla_{A}^{k} \phi\right|_{\tilde{g}}(x) \leq c \tilde{r}^{-k}\left(\frac{\tilde{r}_{0}}{\tilde{r}^{3}}+\frac{1}{\tilde{r}_{1}^{2}}\right)\|\phi\|_{L^{2}(\Omega, \tilde{g})}=c r^{-k} \lambda^{k}\left(\frac{\lambda^{3} r_{0}}{\lambda r^{3}}+\frac{\lambda^{2}}{r_{1}^{2}}\right)\|\phi\|_{L^{2}(\Omega, \tilde{g})} .
$$

Since $\|\phi\|_{L^{2}(\Omega, \tilde{g})}=\lambda^{-2}\|\phi\|_{L^{2}(\Omega, g)}$ and $\left|\nabla_{A}^{k} \phi\right|_{\tilde{g}}=\lambda^{k}\left|\nabla_{A}^{k} \phi\right|_{g}$, we see that

$$
\left|\nabla_{A}^{k} \phi\right|_{g}(x) \leq c\left(\frac{r_{0}}{r^{3}}+\frac{1}{r_{1}^{2}}\right)\|\phi\|_{L^{2}(\Omega, g)}, \quad 2 r_{0} \leq r \leq r_{1} / 2 .
$$

as desired.

Observe that because $r_{0}<r<r_{1}$, our hypotheses (1.2) on the scalar curvature $\kappa_{g}$ and (1.3) on the curvature $F_{A}$ (together with the decay estimates (2.3), (2.6), or (2.8)) imply that for $r_{0} \leq r \leq r_{1}$,

$$
\begin{aligned}
\left|F_{A}\right|_{g}(x) & \leq c \varepsilon\left(r_{0}^{2} r^{-4}+r_{1}^{-2}\right) \leq c \varepsilon r^{-2}, \\
\left|\kappa_{g}\right|(x) & \leq c \varepsilon r^{-2} .
\end{aligned}
$$

Substituting the $r^{-2}$ decay estimates (5.4) and (5.5) for $\left|F_{A}\right|$ and $\kappa_{g}$ on $\Omega\left(x_{0}, r_{0}, r_{1}\right)$ into the differential inequality (4.5), we obtain

$$
\Delta|\phi|^{2 / 3} \leq c \varepsilon r^{-2}|\phi|^{2 / 3} \quad \text { on } \Omega\left(x_{0}, r_{0}, r_{1}\right) .
$$

Set $E=\|\phi\|_{L^{2}\left(\Omega\left(x_{0}, r_{0}, r_{1}\right)\right)}$ and fix an arbitrary $\delta \in(0,1)$. At this point it simplifies matters considerably if we take advantage of Lemma 5.6 and for the remainder of this section, without loss of generality, assume $r_{0} \geq 1$. By choosing an $\varepsilon$ small enough that

$$
12 c \varepsilon \leq \delta(2-\delta),
$$

we see that inequalities (5.2), (5.3), and (5.6) imply

$$
\begin{cases}|\phi|^{2 / 3} \leq c E^{2 / 3} r_{0}^{-4 / 3} & \text { on } \partial B\left(x_{0}, 2 r_{0}\right), \\ |\phi|^{2 / 3} \leq c E^{2 / 3} r_{1}^{-4 / 3} & \text { on } \partial B\left(x_{0}, r_{1} / 2\right), \\ (\Delta-\delta(2-\delta) \xi)|\phi|^{2 / 3} \leq 0 & \text { on } \Omega\left(x_{0}, 2 r_{0}, r_{1} / 2\right) .\end{cases}
$$

(The constraint $\delta<1$ ensures that $-\delta(2-\delta)>-1$ and so Lemma 5.1 applies to the operator $L=\Delta-\delta(2-\delta) \xi$.)

Lemma 5.7. 21, Equation (3.10)] Let $(X, g)$ be a Riemannian, smooth m-manifold, let $h$ be a smooth positive harmonic function on $X$, let $\xi=|d(\log \sqrt{h})|^{2}$, and let $\alpha$ be a constant. Then

$$
\Delta h^{\alpha}=4 \alpha(1-\alpha) \xi h^{\alpha} .
$$

Proof. One simply calculates that

$$
\Delta h^{\alpha}=d^{*} d h^{\alpha}=\alpha(1-\alpha) h^{\alpha}\left|h^{-1} d h\right|^{2}=4 \alpha(1-\alpha) \xi h^{\alpha},
$$

as desired. 
We apply Lemma 5.7 to the harmonic function $h$ on $(X, g)$. We observe that

$$
4 \alpha(1-\alpha)=\delta(2-\delta),
$$

for $\alpha=\delta / 2$ or $\alpha=1-\delta / 2$. Hence, Lemma 5.7 yields

$$
\begin{gathered}
\Delta h^{\delta / 2}=\delta(2-\delta) \xi h^{\delta / 2}, \\
\Delta h^{1-\delta / 2}=\delta(2-\delta) \xi h^{1-\delta / 2} .
\end{gathered}
$$

Therefore, we define

$$
g_{1}=r_{0}^{-\delta+2 / 3} h^{1-\delta / 2}+r_{1}^{\delta-4 / 3} h^{\delta / 2} .
$$

Then the definition of $g_{1}$, the fact that $0<\delta<1$, the inequalities (5.2), and the identities in (5.9) imply that

$$
\begin{cases}g_{1} \geq \frac{1}{2} r_{0}^{-4 / 3} & \text { on } \partial B\left(x_{0}, 2 r_{0}\right), \\ g_{1} \geq \frac{1}{2} r_{1}^{-4 / 3} & \text { on } \partial B\left(x_{0}, r_{1} / 2\right), \\ (\Delta-\delta(2-\delta) \xi) g_{1}=0 & \text { on } \Omega\left(x_{0}, 2 r_{0}, r_{1} / 2\right)\end{cases}
$$

Thus, it follows from Lemma 5.1 that, on $\Omega\left(x_{0}, 2 r_{0}, r_{1} / 2\right)$,

$$
\begin{aligned}
|\phi|^{2 / 3} \leq c E^{2 / 3} g_{1} & =c E^{2 / 3}\left(r_{0}^{-\delta+2 / 3} h^{1-\delta / 2}+r_{1}^{\delta-4 / 3} h^{\delta / 2}\right) \\
& =c E^{2 / 3}\left(r_{0}^{-4 / 3}\left(r_{0}^{2} h\right)^{1-\delta / 2}+r_{1}^{-4 / 3}\left(r_{1}^{2} h\right)^{\delta / 2}\right) .
\end{aligned}
$$

If we now substitute our hypothesized decay estimates (1.2) for $\left|\kappa_{g}\right|$ and the estimate for $\left|F_{A}\right|$ on $\Omega\left(x_{0}, r_{0}, r_{1}\right)$ given by (2.3), (2.6), or (2.8)) (courtesy of $(1.3)$ ), and the preliminary decay estimate (5.11) for $\phi$ into the differential inequality (4.8) for $\Delta|\phi|^{2 / 3}$, we obtain (using $r_{0} \geq 1,2 r_{0}<r<r_{1} / 2, \delta<1$, and inequalities (5.2) for $h$ )

$$
\begin{aligned}
\Delta|\phi|^{2 / 3} & \leq c \varepsilon\left(r_{0}^{2} r^{-4}+r_{1}^{-2}\right)|\phi|^{2 / 3} \quad \text { on } \Omega\left(x_{0}, 2 r_{0}, r_{1} / 2\right) \\
& \leq c \varepsilon\left(r_{0}^{-4 / 3}\left(r_{0}^{2} h\right)^{3-\delta / 2}+r_{1}^{-4 / 3}\left(r_{1}^{2} h\right)^{\delta / 2}\right) .
\end{aligned}
$$

Therefore, substituting the preliminary decay estimate (5.11) for $\phi$ into (5.12), together with the upper bound (5.2) for $r^{-2}$, yields

$$
\begin{cases}|\phi|^{2 / 3} \leq c E^{2 / 3} r_{0}^{-4 / 3} & \text { on } \partial B\left(x_{0}, 2 r_{0}\right), \\ |\phi|^{2 / 3} \leq c E^{2 / 3} r_{1}^{-4 / 3} & \text { on } \partial B\left(x_{0}, r_{1} / 2\right), \\ \Delta|\phi|^{2 / 3} \leq c \varepsilon E^{2 / 3}\left(r_{0}^{-4 / 3}\left(r_{0}^{2} h\right)^{3-\delta / 2}+r_{1}^{-4 / 3}\left(r_{1}^{2} h\right)^{\delta / 2}\right) & \text { on } \Omega\left(x_{0}, 2 r_{0}, r_{1} / 2\right) .\end{cases}
$$

Define

$$
g_{2}=2\left(r_{0}^{2 / 3} h+r_{1}^{-4 / 3}\right)-\left(r_{0}^{-4 / 3}\left(r_{0}^{2} h\right)^{2-\delta / 2}+r_{1}^{-4 / 3}\left(r_{1}^{2} h\right)^{\delta / 2-1}\right),
$$

and note that

$$
g_{2} \leq 2\left(r_{0}^{2 / 3} h+r_{1}^{-4 / 3}\right),
$$

while, using the inequalities (5.2) for $h$, the fact that $2 r_{0}<r<r_{1} / 2$, and $\delta<1$, gives

$$
g_{2} \geq\left(r_{0}^{2 / 3} h+r_{1}^{-4 / 3}\right) \quad \text { on } \Omega\left(x_{0}, 2 r_{0}, r_{1} / 2\right)
$$


Observe that the identity (5.8) yields

$$
\begin{aligned}
& \Delta h^{2-\delta / 2}=-(4-\delta)(2-\delta) \xi h^{2-\delta / 2}, \\
& \Delta h^{\delta / 2-1}=-(4-\delta)(2-\delta) \xi h^{\delta / 2-1} .
\end{aligned}
$$

Then, by definition of $g_{2}$, the lower bound (5.2) for $\xi$, and the preceding inequalities on $\partial \Omega\left(x_{0}, 2 r_{0}, r_{1} / 2\right)$, we see that

$$
\left\{\begin{aligned}
g_{2} \geq & \frac{1}{2} r_{0}^{-4 / 3} & & \text { on } \partial B\left(x_{0}, 2 r_{0}\right), \\
g_{2} \geq & \frac{1}{2} r_{1}^{-4 / 3} & & \text { on } \partial B\left(x_{0}, r_{1} / 2\right), \\
\Delta g_{2} \geq & c(4-\delta)(2-\delta) & & \\
& \times\left(r_{0}^{-4 / 3}\left(r_{0}^{2} h\right)^{3-\delta / 2}+r_{1}^{-4 / 3}\left(r_{1}^{2} h\right)^{\delta / 2}\right) & & \text { on } \Omega\left(x_{0}, 2 r_{0}, r_{1} / 2\right) .
\end{aligned}\right.
$$

Note that the constant $(4-\delta)(2-\delta)$ is positive as we assumed $\delta<1$. Therefore, it follows from the inequalities $(5.13)$ and $(5.16)$ that

$$
\begin{aligned}
|\phi|^{2 / 3} & \leq c E^{2 / 3} g_{2} \text { on } \partial \Omega\left(x_{0}, 2 r_{0}, r_{1} / 2\right), \\
\Delta|\phi|^{2 / 3} & \leq \Delta\left(c \varepsilon E^{2 / 3} g_{2}\right) \leq \Delta\left(c E^{2 / 3} g_{2}\right) \text { on } \Omega\left(x_{0}, 2 r_{0}, r_{1} / 2\right) .
\end{aligned}
$$

Hence, the comparison principle for the Laplacian $\Delta$ and the upper bound (5.14) for $g_{2}$ that on $\Omega\left(x_{0}, 2 r_{0}, r_{1} / 2\right)$ implies that

$$
|\phi|^{2 / 3} \leq c E^{2 / 3} g_{2} \leq c E^{2 / 3}\left(r_{0}^{2 / 3} h+r_{1}^{-4 / 3}\right)
$$

and so

$$
|\phi| \leq c E\left(r_{0} h^{3 / 2}+r_{1}^{-2}\right) \leq c E\left(r_{0} r^{-3}+r_{1}^{-2}\right) .
$$

This completes the proof of Theorem 1.1 when $k=0$. The cases $k \geq 1$ follow from immediately from the case $k=0$ and Lemma 5.5.

5.2. Decay estimates for eigenspinors and proof of Corollary 1.2. We now allow $\phi$ to be an eigenspinor of $D_{A}$, with non-zero eigenvalue $\mu$. Thus $\phi$ is a $D_{\tilde{A}}$-harmonic spinor when $\tilde{A}$ is the Friedrich connection (4.7) defined by $A$ and $\mu$. The curvature $F_{\tilde{A}}$ obeys

$$
\left|F_{\tilde{A}}\right| \leq\left|F_{A}\right|+c \mu^{2}
$$

where $c$ is a universal constant (independent of the metric). We must assume that $\mu^{2}$ satisfies the inequalities (1.2) obeyed by $\kappa_{g}$ for suitable $r_{0} \leq r \leq r_{1}$, so in the argument of $\$ 5.1$ the bounds (5.6) and (5.12) continue to hold with $\left|\kappa_{g}\right|$ replaced by $\left|\kappa_{g}\right|+\mu^{2}$ when $\mu \neq 0$. Writing $\mu^{2}=\mu^{2} r_{1}^{2} r_{1}^{-2}$, one trivially has

$$
\mu^{2} \leq \varepsilon r_{1}^{-2} \leq \varepsilon r^{-2}, \quad r_{0} \leq r \leq r_{1},
$$

provided $r_{1}$ is small enough that $r_{1}^{2} \leq \varepsilon$. Then the inequalities (1.2) hold with $\mu^{2}$ in place of $\kappa_{g}$ when $c_{1}=1$ and $c_{2}=r_{1}^{2}$. Hence, the same argument which proves Theorem 1.1 leads to a proof of Corollary 1.2, since the additional term $F_{\tilde{A}}-F_{A}$ obeys the same estimates (when $r_{1}^{2} \leq \varepsilon$ ) and has the same scaling behavior as $\kappa_{g}$. 


\section{Bubbling and elliptic estimates for the Dirac operator}

Theorem 1.1 and Corollary 1.2 provide pointwise decay estimates for eigenspinors which are useful over annuli in a four-manifold $(X, g)$ where we have suitable bounds on the curvatures of $A$ and the Levi-Civita connection $\nabla_{g}$. In this section we describe (see Proposition 6.1) how one can apply Theorem 1.1 to the problem of obtaining $C^{0} \cap L_{2}^{2}$ estimates for negative harmonic spinors when the induced $\mathrm{SO}(3)$ connection $A$ on $\mathfrak{s u}(E)$ "bubbles" in the Uhlenbeck sense [32]. Estimates of this kind have important applications to the problem of gluing $\mathrm{PU}(2)$ monopoles [16], [17]. We emphasize negative spinors because it is the anti-self-dual component of the curvature which appears in the Bochner formula (4.4) for $D_{A}^{2}$ on negative spinors, whereas the self-dual component of the curvature appears in the Bochner for $D_{A}^{2}$ on positive spinors: with the usual conventions of gauge theory (for the anti-self-dual Yang-Mills equation [9] or the PU(2) monopole equations [12]), the anti-selfdual component of the curvature can bubble while one has good control on the self-dual component. Some remarks on the significance of Proposition 6.1 are included in $\$ 6.2$.

6.1. Integral estimates for negative spinors when the anti-self-dual curvature component bubbles. To describe our application, we continue with the setup of $\$ 1.1$ and write $V=V^{+} \oplus V^{-}$, where $V^{ \pm}=W^{ \pm} \otimes E$. We further suppose that $E$ is complex rank two, let $\hat{A}$ denote the induced $\mathrm{SO}(3)$ connection on $\mathfrak{s u}(E)$, and let $A_{e}$ denote the induced unitary connection on the line bundle $\operatorname{det}(E)$. In gauge-theoretic applications [9], [12], it is the curvature component $F_{\hat{A}}$ which may bubble, while the remaining connections (on $W$ and $\operatorname{det}(E))$ are usually fixed. We allow the constant $c$ in our estimates below to depend on $C^{2}$ bounds on the curvature of the Riemannian metric $g$ and the connections $A_{d}$ and $A_{e}$.

Recall [10, §4], [31] that the $L^{\sharp}$ and $L^{2 \sharp}$ Sobolev norms are defined by

$$
\begin{aligned}
\|\phi\|_{L^{\sharp}(X)} & :=\sup _{x \in X}\left\|\operatorname{dist}^{-2}(x, \cdot)|\phi|\right\|_{L^{1}(X)} \quad \text { and } \quad\|\phi\|_{L^{\sharp, 2}(X)}:=\|\phi\|_{L^{\sharp}(X)}+\|\phi\|_{L^{2}(X)} . \\
\|\phi\|_{L^{2 \sharp}(X)} & :=\sup _{x \in X}\left\|\operatorname{dist}^{-1}(x, \cdot)|\phi|\right\|_{L^{2}(X)} \quad \text { and } \quad\|\phi\|_{L^{2 \sharp, 4}(X)}:=\|\phi\|_{L^{2 \sharp}(X)}+\|\phi\|_{L^{4}(X)} .
\end{aligned}
$$

See [10, §4], [31] for further explanation and properties of this family of Sobolev norms.

Proposition 6.1. Let $X$ be a closed, oriented four-manifold with metric g. Given $m \in \mathbb{N}$, there are positive constants $r_{1}, c\left(m, r_{1}\right)$, and $\varepsilon$ such that for small enough $\varepsilon$ the following holds. Suppose $\left\{x_{i}\right\}_{i=1}^{m}$ is a finite set of distinct points and $\left\{\lambda_{i}\right\}_{i=1}^{m} \subset\left(0, r_{1}\right]$ is a set of positive constants. Let

$$
U=X-\bigcup_{i=1}^{m} B\left(x_{i}, \frac{1}{2} \lambda_{i}^{1 / 2}\right) \quad \text { and } \quad U^{\prime}=X-\bigcup_{i=1}^{m} B\left(x_{i}, 4 \lambda_{i}^{1 / 3}\right) \Subset U .
$$

Assume the $L^{2}$ norms of the curvatures of $A_{d}, A_{e}$, and $\hat{A}$ on the annuli $\Omega\left(x_{i}, \frac{1}{2} \lambda_{i}^{1 / 3}, r_{1}\right)$ are less than or equal to $\varepsilon$. If $\phi \in C^{\infty}\left(X, V^{-}\right) \cap \operatorname{Ker} D_{A}$, then

$$
\|\phi\|_{C^{0} \cap L_{2, A}^{2}\left(U^{\prime}\right)} \leq c\left(1+\left\|F_{\hat{A}}\right\|_{L^{2}(X)}\right)\left(1+\left\|F_{\hat{A}}^{-}\right\|_{C^{0}(U)}\right)^{2}\|\phi\|_{L^{2}(U)} .
$$

In a typical application of of Proposition 6.1, the curvature of $\hat{A}$ would be allowed to bubble near the points $x_{i} \in X$. Before proceeding with the proof, we recall the following important estimate from [10]:

Lemma 6.2. 10, Lemma 5.5] Let $X$ be a closed, oriented, Riemannian four-manifold. Then there is a constant $c$ with the following significance. Let $\mathcal{F}$ be a Riemannian vector 
bundle over $X$ and let $B$ be an orthogonal $C^{\infty}$ connection on $\mathcal{F}$ with curvature $F_{B}$. Then the following estimate holds for any $\phi \in C^{\infty}(\mathcal{F})$ :

$$
\|\phi\|_{C^{0} \cap L_{2, B}^{2}(X)} \leq c\left(1+\left\|F_{B}\right\|_{L^{2}(X)}\right)\left(\left\|\nabla_{B}^{*} \nabla_{B} \phi\right\|_{L^{\sharp, 2}(X)}+\|\phi\|_{L^{2}(X)}\right) .
$$

Proof of Proposition 6.1. We choose a smooth cutoff function $\beta$ so that (see the proof of Lemma 5.8 in 14])

$$
\|\nabla \beta\|_{L^{2 \sharp, 4}(X)}+\left\|\nabla^{2} \beta\right\|_{L^{\sharp, 2}(X)} \leq c,
$$

for some constant $c(g)$, where

$$
\beta= \begin{cases}1 & \text { on } X-\cup_{i=1}^{m} B\left(x_{i}, 2 \lambda_{i}^{1 / 3}\right), \\ 0 & \text { on } \cup_{i=1}^{m} B\left(x_{i}, \lambda_{i}^{1 / 3}\right) .\end{cases}
$$

For the bound on $\left\|\nabla^{2} \beta\right\|_{L^{\sharp}(X)}$ one uses the fact that this norm is scale invariant (it has the same scaling behavior as $\left.\left\|\nabla^{2} \beta\right\|_{L^{2}(X)}\right)$, so we can assume that $\lambda_{i}=1$ without loss of generality when deriving the uniform bound on $\left\|\nabla^{2} \beta\right\|_{L^{\sharp}(X)}$.

Note that $\beta=1$ on $U^{\prime}$ and $\operatorname{supp} \beta \subset U$. Schematically, we have

$$
\nabla_{A}^{*} \nabla_{A}(\beta \phi)=(\Delta \beta) \phi+\nabla \beta \otimes \nabla_{A} \phi+\beta \nabla_{A}^{*} \nabla_{A} \phi,
$$

and therefore the cutoff function derivative bounds (6.1) yield

$$
\begin{aligned}
\left\|\nabla_{A}^{*} \nabla_{A}(\beta \phi)\right\|_{L^{\sharp, 2}(X)} \leq & c\|\phi\|_{C^{0}(\operatorname{supp} d \beta)}+c\left\|\nabla_{A} \phi\right\|_{L^{2 \sharp, 4}(\operatorname{supp} d \beta)} \\
& +\left\|\nabla_{A}^{*} \nabla_{A} \phi\right\|_{L^{\sharp, 2}(\operatorname{supp} \beta)} .
\end{aligned}
$$

Since $D_{A}^{2} \phi=0$, the Bochner formula (4.4) implies that

$$
\left\|\nabla_{A}^{*} \nabla_{A} \phi\right\|_{L^{\sharp, 2}(\operatorname{supp} \beta)} \leq c\left(1+\left\|F_{\hat{A}}^{-}\right\|_{C^{0}(\operatorname{supp} \beta)}\right)\|\phi\|_{L^{\sharp, 2}(\operatorname{supp} \beta)} .
$$

Theorem 1.1 gives a $C^{0}$ bound for $\phi$ on $\operatorname{supp} d \beta \subset \cup_{i=1}^{m} \Omega\left(x_{i}, \lambda_{i}^{1 / 3}, 2 \lambda_{i}^{1 / 3}\right)$ :

$$
\|\phi\|_{C^{0}(\operatorname{supp} d \beta)} \leq \sum_{i=1}^{m}\|\phi\|_{C^{0}\left(\Omega\left(x_{i}, \lambda_{i}^{1 / 3}, 2 \lambda_{i}^{1 / 3}\right)\right)} \leq c m\|\phi\|_{L^{2}(U)},
$$

where we take $r_{0}=\lambda_{i}$. Lemma 4.1 in 10 implies that $\|\phi\|_{L^{\sharp}(\operatorname{supp} \beta)} \leq c\|\phi\|_{L^{4}(\operatorname{supp} \beta)}$. We may consider $U$ as the union of the region $U_{0}=X-\cup_{i=1}^{m} B\left(x_{i}, \frac{1}{2} r_{1}\right)$ and the annuli $\Omega\left(x_{i}, \lambda_{i}^{1 / 3}, r_{1}\right)$. We can choose a cutoff function $\chi$ which is equal to one $U_{0}$ and supported on the complement of the balls $B\left(x_{i}, \frac{1}{4} r_{1}\right)$. We now apply the Sobolev embedding $L_{1, A}^{2} \subset L^{4}$ (with embedding constant independent of $A$ ), integration by parts, and the Bochner formula (4.4) to obtain

$$
\|\chi \phi\|_{L^{4}(X)} \leq c\|\chi \phi\|_{L_{1, A}^{2}(X)} \leq c\left(1+\left\|F_{\hat{A}}^{-}\right\|_{C^{0}\left(U_{0}\right)}\right)\|\chi \phi\|_{L^{2}(X)} .
$$

Over the annuli $\Omega\left(x_{i}, \lambda_{i}^{1 / 3}, r_{1}\right)$, Theorem 1.1 yields bounds of the form

$$
\|\phi\|_{L_{1, A}^{2}\left(\Omega\left(x_{i}, \lambda_{i}^{1 / 3}, r_{1}\right)\right)} \leq c\|\phi\|_{L^{2}(U)} .
$$

Hence, combining these inequalities, we have

$$
\|\phi\|_{L^{\sharp}(\operatorname{supp} \beta)} \leq c\left(1+\left\|F_{\hat{A}}^{-}\right\|_{C^{0}\left(U_{0}\right)}\right)\|\phi\|_{L^{2}(U)} .
$$


We next consider the term $\left\|\nabla_{A} \phi\right\|_{L^{4}(\operatorname{supp} d \beta)}$ on the right-hand side of estimate (6.2). Denoting $C_{1}=\|\phi\|_{L^{2}(U)}$, we observe that Theorem 1.1 (again taking $r_{0}=\lambda_{i}$ ) gives

$$
\begin{aligned}
\left\|\nabla_{A} \phi\right\|_{L^{4}(\operatorname{supp} d \beta)}^{4} & =c C_{1}^{4} \sum_{i=1}^{m} \int_{\lambda_{i}^{1 / 3}}^{2 \lambda_{i}^{1 / 3}} r^{-4}\left(\lambda_{i} r^{-3}+r_{1}^{2}\right)^{4} r^{3} d r \\
& \leq c C_{1}^{4} \sum_{i=1}^{m} \int_{\lambda_{i}^{1 / 3}}^{2 \lambda_{i}^{1 / 3}} r^{-1} d r \\
& =\left.c C_{1}^{4} \sum_{i=1}^{m} \log r\right|_{\lambda_{i}^{1 / 3}} ^{2 \lambda_{i}^{1 / 3}}=c m C_{1}^{4} \log 2,
\end{aligned}
$$

and therefore

$$
\left\|\nabla_{A} \phi\right\|_{L^{4}(\operatorname{supp} d \beta)} \leq c\|\phi\|_{L^{2}(U)} \text {. }
$$

For the term $\left\|\nabla_{A} \phi\right\|_{L^{2 \sharp}(\operatorname{supp} d \beta)}$ on the right-hand side of estimate (6.2), choose a cutoff function $\gamma$ defined in much the same way as $\beta$, except that $\gamma=0$ on the complement of the annulus $\Omega\left(x_{i}, \frac{1}{2} \lambda_{i}^{1 / 3}, 4 \lambda_{i}^{1 / 3}\right)$ and $\gamma=1$ on the annulus $\Omega\left(x_{i}, \lambda_{i}^{1 / 3}, 2 \lambda_{i}^{1 / 3}\right)$. Thus, we have $\operatorname{supp} \gamma \subset U$. Then

$$
\begin{aligned}
\left\|\nabla_{A} \phi\right\|_{L^{2 \sharp}(\operatorname{supp} d \beta)} & \left.\leq\left\|\gamma \nabla_{A} \phi\right\|_{L^{2 \sharp}(X)} \leq\left\|\gamma \nabla_{A} \phi\right\|_{L_{1, A}^{2}(X)} \quad \text { (Lemma } 4.1 \text { in } 10 \|\right) \\
& \leq c\left\|\gamma \nabla_{A} \phi\right\|_{L^{4}(X)}+\left\|\nabla_{A}\left(\gamma \nabla_{A} \phi\right)\right\|_{L^{2}(X)} \\
& \leq c\left(\left\|\nabla_{A} \phi\right\|_{L^{4}(\operatorname{supp} \gamma)}+\left\|\nabla_{A}^{2} \phi\right\|_{L^{2}(\operatorname{supp} \gamma)}\right) .
\end{aligned}
$$

The final inequality above follows from Hölder's inequality and the uniform $L^{4}$ bound on $\nabla \gamma$, analogous to that in (6.1) for $\nabla \beta$. The argument yielding inequality (6.6) also provides a similar bound on $\left\|\nabla_{A} \phi\right\|_{L^{4}(\operatorname{supp} \gamma)}$. For the term $\left\|\nabla_{A}^{2} \phi\right\|_{L^{2}(\operatorname{supp} \gamma)}$, Theorem 1.1 gives

$$
\begin{aligned}
\left\|\nabla_{A}^{2} \phi\right\|_{L^{2}(\operatorname{supp} \gamma)}^{2} & =c C_{1}^{2} \sum_{i=1}^{m} \int_{\frac{1}{2} \lambda_{i}^{1 / 3}}^{4 \lambda_{i}^{1 / 3}} r^{-4}\left(\lambda_{i} r^{-3}+r_{1}^{2}\right)^{2} r^{3} d r \\
& \leq c C_{1}^{2} \sum_{i=1}^{m} \int_{\frac{1}{2} \lambda_{i}^{1 / 3}}^{4 \lambda_{i}^{1 / 3}} r^{-1} d r \\
& =c m C_{1}^{2} \log 2,
\end{aligned}
$$

and therefore, combining estimates (6.6) (now for $\left\|\nabla_{A} \phi\right\|_{L^{4}(\operatorname{supp} \gamma)}$ ) and (6.7) with the preceding inequality, we obtain

$$
\left\|\nabla_{A} \phi\right\|_{L^{2 \sharp}(\operatorname{supp} d \beta)} \leq c\|\phi\|_{L^{2}(U)} .
$$

Combining the inequalities $(6.2),(6.3),(6.4),(6.5),(6.6)$, and 6.8) gives

$$
\left\|\nabla_{A}^{*} \nabla_{A}(\beta \phi)\right\|_{L^{\sharp, 2}(X)} \leq c\left(1+\left\|F_{\hat{A}}^{-}\right\|_{C^{0}(U)}\right)^{2}\|\phi\|_{L^{2}(U)} .
$$

Substituting the preceding bound into the estimate given by Lemma 6.2 for $\beta \phi$ yields

$$
\begin{aligned}
\|\phi\|_{C^{0} \cap L_{2, A}^{2}\left(U^{\prime}\right)} & \leq\|\beta \phi\|_{C^{0} \cap L_{2, A}^{2}(X)} \\
& \leq c\left(1+\left\|F_{\hat{A}}\right\|_{L^{2}(X)}\right)\left(1+\left\|F_{\hat{A}}^{-}\right\|_{C^{0}(U)}\right)^{2}\|\phi\|_{L^{2}(U)} .
\end{aligned}
$$

This completes the proof. 
6.2. Remarks on the result. In contrast to the proof of Proposition 6.1, which relied on our pointwise decay estimates for negative harmonic spinors, the Bochner formula (4.4) and a simple integration-by-parts argument yields a useful estimate for positive harmonic spinors:

Lemma 6.3. Let $X$ be a closed, oriented, Riemannian four-manifold. Then there are positive constants $c, \varepsilon$ so that for small enough $\varepsilon$ the following holds. Suppose $X=U_{2} \cup U_{\infty}$, where $U_{2} \Subset X$ is an open subset with $\left\|F_{\hat{A}}^{+}\right\|_{L^{\sharp, 2}\left(U_{2}\right)}<\varepsilon$. If $\phi \in C^{\infty}\left(X, V^{+}\right) \cap \operatorname{Ker} D_{A}$, then

$$
\|\phi\|_{C^{0} \cap L_{2, A}^{2}(X)} \leq c\left(1+\left\|F_{\hat{A}}\right\|_{L^{2}(X)}\right)^{2}\left(1+\left\|F_{\hat{A}}^{+}\right\|_{C^{0}\left(U_{\infty}\right)}\right)^{2}\|\phi\|_{L^{2}(X)} .
$$

Proof. From the Bochner formula (4.4) and the Sobolev multiplication [10, Lemma 4.3],

$$
\left\|\nabla_{A}^{*} \nabla_{A} \phi\right\|_{L^{\sharp, 2}} \leq c\left(1+\left\|F_{\hat{A}}^{+}\right\|_{C^{0}\left(U_{\infty}\right)}\right)\|\phi\|_{L^{\sharp, 2}}+c\left\|F_{\hat{A}}^{+}\right\|_{L^{\sharp, 2}\left(U_{2}\right)}\|\phi\|_{C^{0}\left(U_{2}\right)} .
$$

Combining the preceding estimate with that of Lemma 6.2, together with the embedding and interpolation inequalities $\|\phi\|_{L^{\sharp}} \leq c\|\phi\|_{L^{4}} \leq c\|\phi\|_{L^{2}}^{1 / 2}\|\phi\|_{C^{0}}^{1 / 2}$, choosing $\varepsilon$ small enough so that $c\left\|F_{\hat{A}}^{+}\right\|_{L^{\sharp, 2}\left(U_{2}\right)} \leq \frac{1}{2}$, and using rearrangement with the terms involving $\|\phi\|_{C^{0}}$ then yields the desired bound.

Lemma 6.3 provides useful $C^{0} \cap L_{2}^{2}(X)$ elliptic estimates for positive spinors $\phi \in C^{\infty}\left(X, V^{+}\right)$ even when $\hat{A}$ bubbles, because we still have uniform $C^{0}$ bounds for $F_{\hat{A}}^{+}$away from the bubble points (on the set $U_{\infty}$ ) and small $L^{\sharp, 2}$ bounds around the bubble points (on the set $U_{2}$ ). However, this is never the case for $F_{\hat{A}}^{-}$in such applications because $F_{\hat{A}}^{-}$is neither $C^{0}$-bounded nor $L^{\sharp, 2}$-small around the bubble points. So the analogue of Lemma 6.3 for negative spinors - which would have $F_{\hat{A}}^{-}$in place of $F_{\hat{A}}^{+}$- does not provide useful $C^{0} \cap L_{2}^{2}(X)$ elliptic estimates for $\phi \in C^{\infty}\left(X, V^{-}\right)$. In contrast, in Proposition 6.1 where $U$ plays the role of $U_{\infty}$ above - we at least still obtain an estimate for $\phi$ on the complement of the small balls $B\left(x_{i}, 4 \lambda_{i}^{1 / 3}\right)$, which is uniform with respect $\lambda_{i}$ as those constants tend to zero.

\section{REFERENCES}

[1] T. Aubin, Nonlinear analysis on complex manifolds. Monge-Ampère equations, Springer, New York, 1982.

[2] S. Bando, A. Kasue, and H. Nakajima, On a construction of coordinates at infinity on manifolds with fast curvature decay and maximal volume growth, Invent. Math. 97 (1989), 313-349.

[3] C. Bär, The Dirac operator on space forms of positive curvature, J. Math. Soc. Japan 48 (1996), 69-83.

[4] J-P. Bourguignon, The "magic" of Weitzenböck formulas, Variational methods, Proceedings of the Conference on Variational Problems, Paris, 1988 (H. Berestycki, J-M. Coron, and I. Ekeland, eds.), Progress in nonlinear differential equations and their applications, vol. 4, Birkhäuser, Boston, MA, 1990, pp. 251-271.

[5] T. Branson, Kato constants in Riemannian geometry, Math. Res. Lett. 7 (2000), 245-261, preprint version at http://www.math.uiowa.edu/ branson.

[6] D. M. J. Calderbank, P. Gauduchon, and M. Herzlich, Refined Kato inequalities and conformal weights in Riemannian geometry, J. Funct. Anal. 173 (2000), 214-255, math.DG/9909116.

[7] On the Kato inequality in Riemannian geometry, preprint.

[8] S. K. Donaldson, An application of gauge theory to four-dimensional topology, J. Differential Geom. 18 (1983), 279-315.

[9] S. K. Donaldson and P. B. Kronheimer, The geometry of four-manifolds, Oxford Univ. Press, Oxford, 1990.

[10] P. M. N. Feehan, Critical-exponent norms and the slice theorem for the quotient space of connections, Pac. J. Math., to appear; dg-ga/9711004 (v2). 
[11] _ A Kato-Yau inequality for harmonic spinors and decay estimate for eigenspinors, preliminary version; math.DG/9903021 (v3).

[12] P. M. N. Feehan and T. G. Leness, PU(2) monopoles. I: Regularity, Uhlenbeck compactness, and transversality, J. Differential Geom. 49 (1998), 265-410, dg-ga/9710032.

[13] _ PU(2) monopoles and relations between four-manifold invariants, Topology Appl. 88 (1998), 111-145, dg-ga/9709022

[14] _ Donaldson invariants and wall-crossing formulas. I: Continuity of gluing and obstruction maps, submitted to a print journal, math.DG/9812060 (v3).

[15] _ PU(2) monopoles. II: Top-level Seiberg-Witten moduli spaces and Witten's conjecture in low degrees, submitted to a print journal, dg-ga/9712005.

[16] , PU(2) monopoles. III: Existence of gluing and obstruction maps, submitted to a print journal; math.DG/9907107.

[17] , PU(2) monopoles. IV: Surjectivity of gluing maps, in preparation.

[18] D. Freed and K. K. Uhlenbeck, Instantons and four-manifolds, 2nd ed., Springer, New York, 1991.

[19] Th. Friedrich, Der erste Eigenwert des Dirac-Operators einer kompakten, Riemannschen Mannigfaltigkeit nichtnegativer Skalarkrümmung, Math. Nachr. 97 (1980), 117-146.

[20] D. Gilbarg and N. Trudinger, Elliptic partial differential equations of second order, second ed., Springer, New York, 1983.

[21] D. Groisser and T. H. Parker, Sharp decay estimates for Yang-Mills fields, Comm. Anal. Geom. 5 (1997), 439-474.

[22] N. Hitchin, Harmonic spinors, Adv. in Math. 14 (1974), 1-55.

[23] M. Itoh and H. Nakajima, Yang-Mills connections and Einstein-Hermitian metrics, Kähler metric and moduli spaces (T. Ochiai, ed.), Advanced Studies in Pure Mathematics, vol. 18-II, Academic Press, Boston, MA, 1990, pp. 395-457.

[24] H. B. Lawson and M-L. Michelsohn, Spin geometry, Princeton Univ. Press, Princeton, NJ, 1988.

[25] H. Nakajima, Yau's trick, Sûgaku 41 (1989), 253-258, (in Japanese; English summary in MR 91j:58173).

[26] _ Moduli spaces of anti-self-dual connections on ALE gravitational instantons, Invent. Math. 102 (1990), 267-303.

[27] J. Råde, Decay estimates for Yang-Mills fields: Two simplified proofs, Global analysis in modern mathematics (Orono, ME, 1991; Waltham, MA 1992), Publish or Perish, Houston, TX, 1993, pp. 91-105.

[28] D. Salamon, Spin geometry and Seiberg-Witten invariants, Birkhäuser, Boston, to appear.

[29] R. Schoen, L. Simon, and S. T. Yau, Curvature estimates for minimal hypersurfaces, Acta Math. 134 (1975), 275-288.

[30] E. Stein, Singular integral operators and differentiability properties of functions, Princeton Univ. Press, Princeton, NJ, 1970.

[31] C. H. Taubes, The stable topology of self-dual moduli spaces, J. Differential Geom. 29 (1989), 162-230.

[32] K. K. Uhlenbeck, Connections with $L^{p}$ bounds on curvature, Comm. Math. Phys. 83 (1982), 31-42.

[33] _ Removable singularities in Yang-Mills fields, Comm. Math. Phys. 83 (1982), 11-29.

[34] D. Yang, Private communication, March 1999.

[35] S-T. Yau, On the Ricci curvature of a compact Kähler manifold and the complex Monge-Ampère equation. I, Comm. Pure Appl. Math. 31 (1978), 339-411.

Department of Mathematics, Ohio State University, Columbus, OH 43210, U.S.A.

E-mail address: feehan@math.ohio-state.edu

URL: http://www .math.ohio-state.edu/ feehan/

Current address: Max Planck Institut für Mathematik, Vivatsgasse 7, Bonn, D-53111, Germany

E-mail address: feehan@mpim-bonn.mpg.de 